\title{
Retinocoroidite toxoplásmica em pacientes com AIDS e neurotoxoplasmose
}

\section{Toxoplasmic retinochoroiditis in patients with AIDS and neurotoxoplasmosis}

\author{
Jane Mary Alves ${ }^{1}$ \\ Vera Magalhães ${ }^{2}$ \\ Marcus Augusto Gomes de Matos ${ }^{3}$
}

Pesquisa realizada nos Hospitais Correa Picanço, Hospital das Clínicas da Universidade Federal de Pernambuco e Hospital Universitário Oswaldo Cruz da Universidade de Pernambuco - Recife (PE) - Brasil.

${ }^{1}$ Pós-graduanda (Mestrado) da Universidade Federal de Pernambuco - UFPE - Recife (PE) - Brasil; Oftalmologista do Instituto de Olhos de Recife - Recife (PE) Brasil.

${ }^{2}$ Doutora, Professora Titular de Doenças Infecciosas e Parasitárias da UFPE - Recife (PE) - Brasil.

${ }^{3}$ Médico do Real Visão do Hospital Português - Recife (PE) - Brasil.

Endereço para correspondência: Jane Mary Alves. Rua Dom Sebastião Leme, 57 - Apto. 202 - Recife (PE) CEP 52011-160

E-mail: janealves.alves@bol.com.br

Recebido para publicação em 25.05.2009

Última versão recebida em 31.12.2009

Aprovação em 11.03.2010

\section{RESUMO}

Objetivo: A neurotoxoplasmose é a alteração do sistema nervoso central mais frequente observada em pacientes com AIDS. A ocorrência de toxoplasmose ocular em neurotoxoplasmose ainda é pouco estudada. O objetivo deste estudo foi de investigar a ocorrência de retinocoroidite toxoplásmica, típica ou provável, em pacientes com AIDS e neurotoxoplasmose. Métodos: Foi desenvolvido estudo prospectivo, tipo série de casos incluindo 70 pacientes, de ambos os sexos, com idade variando de 20 a 63 anos, internados nas enfermarias de três hospitais públicos da cidade do Recife, Pernambuco, com tais diagnósticos firmados segundo os critérios do CDC (1992), no período de janeiro a outubro de 2008. Os pacientes caracterizavam-se por: primeiro episódio de neurotoxoplasmose $(65 ; 92,9 \%)$ ou recidiva $(5 ; 7,1 \%)$; desconhecimento de ter AIDS $(23 ; 32,9 \%)$, contagem média de linfócitos T CD4 de $139,8 \pm 3,04$ células $/ \mathrm{mm}^{3}$ e carga viral média igual a $137.080 \pm 39.380$ cópias/mL. Todos os pacientes foram submetidos a exame oftalmológico, consistindo de: inspeção ocular; aferição da acuidade visual; investigação da função muscular extrínseca ocular e fundoscopia, empregando oftalmoscópio indireto binocular (modelo OHN 3.5 (Eyetec ${ }^{\circledR}$ ) e lente externa de 20 dioptrias $\left(V\right.$ llk $\left.^{\circledR}\right)$. Resultados: Foram diagnosticados $4(5,7 \%)$ pacientes com lesões cicatriciais de retinocoroidite, características de toxoplasmose ocular, sendo típica em $3(75 \%)$ pacientes e bilateral em 1. Não houve qualquer caso de retinocoroidite ativa, típica ou provável. As lesões oculares ativas foram raras comparadas às lesões cicatriciais, as quais se associam à presença de cistos na retina. Conclusão: Recomenda-se que mesmo lesões cicatriciais sejam valorizadas em pacientes com AIDS.

Descritores: Toxoplasmose ocular; Síndrome de imunodeficiência adquirida; Olho/imunologia

\section{INTRODUÇÃO}

A toxoplasmose ocular é a causa mais comum de retinocoroidite em paciente não imunocomprometido, sendo responsável por $30 \%$ a $50 \%$ de todas as uveítes posteriores ${ }^{(1)}$.

Em pacientes com AIDS, a toxoplasmose do sistema nervoso central é a causa mais frequente de lesão cerebral focal ${ }^{(2)}$, principalmente quando a contagem de linfócitos T CD4+ é menor que 100 células $/ \mathrm{mm}^{3(3)}$, e a retinocoroidite toxoplásmica, uma das mais importantes inflamações oculares secundárias ${ }^{(4)}$. É a terceira retinopatia infecciosa mais frequente e corresponde a $1 \%$ a $3 \%$ das infecções oculares nesses pacientes ${ }^{(5-6)}$. 
Lesões oculares podem ser a primeira manifestação de doença intracraniana ou disseminada, porém podem ocorrer sem evidências desse envolvimento ${ }^{(7)}$. Aproximadamente metade dos casos de pacientes com AIDS e toxoplasmose ocular apresenta concomitantemente manifestações de acometimento neurológico. Em contrapartida, os pacientes com doença neurológica pela toxoplasmose têm $10 \%$ a $20 \%$ de chance de apresentar o quadro ocular ${ }^{(8)}$.

$\mathrm{Na}$ AIDS, a retinocoroidite toxoplásmica caracteriza-se geralmente pela presença de lesões branco-floconosas, com quase nenhuma inflamação vítrea ou hemorragia retiniana, sendo as lesões cicatriciais pouco pigmentadas ${ }^{(9-10)}$. Apresentações atípicas, comuns em pacientes com AIDS, imunossupressão iatrogênica ou idade avançada, podem assumir forma bilateral, extensiva ou multifocal ${ }^{(11)}$. Esse aspecto difere da lesão ativa, típica, caracterizada por foco solitário, exsudativo, necrosante de retinocoroidite, adjacente a uma lesão cicatricial, acompanhada de reação vítrea inflamatória moderada a grave, dos pacientes imunocompetentes ${ }^{(12)}$.

Duas pesquisas retrospectivas, realizadas em unidades terciárias de referência em doenças oculares e AIDS, buscaram descrever o comportamento ocular associado à neurotoxoplasmose. Na primeira, envolvendo 145 prontuários, os pacientes foram classificados em dois grupos, segundo tempo decorrido entre o aparecimento das lesões oculares e o tratamento para neurotoxoplasmose. No grupo submetido a tratamento por mais de três meses, $8 \%$ dos pacientes com neurotoxoplasmose tiveram diagnóstico de retinocoroidite toxoplásmica, enquanto que, no grupo com menos de três meses de tratamento, esse porcentual igualou-se a $11,1 \%{ }^{(9)}$. Estudo realizado no Brasil ${ }^{(13)}$ a partir da análise de 118 prontuários permitiu determinar que em $23 \%$ dos casos havia associação entre toxoplasmose ocular e neurotoxoplasmose.

As evidências apontam para a concomitância de atividade da doença nas lesões do SNC e da retina, já que ambos os tecidos têm a mesma origem embriológica e são considerados sítios preferenciais de latência do parasita, mas os dois únicos estu$\operatorname{dos}^{(9,13)}$ que determinaram essa frequência foram discrepantes, tiveram caráter retrospectivo e foram realizados em centros de referência para doença ocular em paciente com AIDS.

Motivados pela possibilidade de essa frequência ser menor que a referida por esses autores, quando realizada prospectivamente em centros de referência para atendimento a pacientes com AIDS, mas não para doenças oculares, buscou-se realizar a presente pesquisa.

O objetivo deste estudo é investigar a ocorrência de retinocoroidite típica ou provável, presumivelmente toxoplásmica, em pacientes com neurotoxoplasmose e AIDS, internados em três serviços de referência para HIV/AIDS.

\section{MÉTODOS}

No período de janeiro a outubro de 2008, foi desenvolvido estudo prospectivo tipo série de casos envolvendo 70 pacientes, com idade igual ou maior que 13 anos, internados nas enfermarias de três hospitais públicos da cidade do Recife, Pernambuco (Hospital Correa Picanço, Hospital das Clínicas da Universidade Federal de Pernambuco e Hospital Universitário Oswaldo Cruz da Universidade de Pernambuco), com diagnóstico de AIDS e acometimento do sistema nervoso central pelo Toxoplasma gondii, firmado segundo os critérios do $\mathrm{CDC}^{(14)}$.

Foram excluídos os pacientes com história de alergia a colírio midriático ou câmara ocular anterior rasa, pelo risco de crise de glaucoma agudo.

A caracterização dos pacientes foi obtida por meio de consulta ao prontuário e compreendeu: dados sociodemográficos, comorbidades oculares, contagem de linfócitos T CD4 e carga viral em sangue periférico.

Todos os pacientes tinham sorologia positiva para HIV e para toxoplasmose (IgG).

O diagnóstico do acometimento do sistema nervoso central pelo Toxoplasma gondii foi realizado com base em manifestações neurológicas clínicas, achados aos exames de imagem e melhora clínica com o tratamento específico instituído.

O comprometimento neurológico grave de todos os pacientes, exigindo que permanecessem em posição supina, fez com que o exame oftalmológico fosse realizado à beira do leito. O exame consistiu em inspeção ocular com lanterna, dirigindo o feixe luminoso paralelamente ao ponto de junção palpebral externo, para avaliação da profundidade da câmara anterior e fundoscopia, após instilação no saco conjuntival de cada olho de 1 gota de colírio de tropicamida a $1 \%$, a cada 10 minutos, por três vezes, para promover midríase. Empregou-se oftalmoscópio indireto binocular (OBI), com iluminação elétrica halógena, modelo OHN 3.5 (Eyetec $^{\circledR}$ ), e lente externa de 20 dioptrias $\left(\right.$ Volk $\left.^{\circledR}\right)$.

Os pacientes com diagnóstico de alterações fundoscópicas foram submetidos a: retinografia, empregando retinógrafo digital (Topcon ${ }^{\circledR}$, Japão), avaliada pelo programa Image NET, para processamento das imagens obtidas, assim como angiofluoresceinografia, quando indicada, após infusão endovenosa periférica rápida de $5 \mathrm{~mL}$ de solução de fluoresceína sódica a $10 \%$.

Todos os exames fundoscópicos foram avaliados por um oftalmologista, o qual acompanhou, juntamente com a pesquisadora, os pacientes que necessitaram de tratamento oftalmológico. Foram afastados os diagnósticos oculares diferenciais como sífilis e infecção por citomegalovírus e herpes.

A retinocoroidite foi classificada por dois sistemas: atividade e tipicidade. Quanto à atividade, a retinocoroidite foi considerada: cicatricial, na presença de margens bem delimitadas, vários graus de hiperplasia do epitélio pigmentário e atrofia retinocoroidea; ou ativa, quando foi possível observar lesão branco-amarelada, ocasionalmente cinza, com limites mal definidos devido ao edema retiniano circunjacente. Quanto à tipicidade, a lesão foi classificada como: típica quando cicatrizada ou ativa, satélite a uma ou mais lesões cicatrizadas, ou provável, quando necrosante, focal, típica, porém isolada, podendo estar em atividade ou cicatrizada. 
Os dados foram organizados com o programa Excel $^{\circledR}$ e resumidos por meio de medidas de posição e de dispersão, apresentados em tabelas.

A pesquisa foi aprovada pelo Comitê de Ética em Pesquisa com Seres Humanos da Universidade Federal de Pernambuco, sob nº 010/2008-CEP/CCS, e CEP/CCS/UFPE № $371 / 07$.

\section{RESULTADOS}

Dentre os 70 pacientes com toxoplasmose do sistema nervoso central, houve predomínio do gênero masculino $(51 ; 72,9 \%)$. A idade variou de 20 a 63 anos. Foram mais frequentes pacientes solteiros (44; 62,9\%) e procedência da cidade de Recife ou de outras da Região Metropolitana (58; 82,9\%) (Tabela 1).

Na tabela 2, são apresentadas as variáveis relativas ao uso de terapêutica medicamentosa, na qual se observa que $16(22,9 \%)$ pacientes relataram fazer uso de terapia antirretroviral com periodicidade irregular. Dentre os 70 pacientes, 65 (92,9\%) não tinham história prévia de neurotoxoplasmose, enquanto que para $5(7,1 \%)$, ocorreu como recidiva da doença. Os cinco pa-

\begin{tabular}{|c|c|c|}
\hline Variáveis sociodemográficas & Frequência & Porcentual (\%) \\
\hline \multicolumn{3}{|l|}{ Gênero } \\
\hline Masculino & 51 & 72,9 \\
\hline Feminino & 19 & 27,1 \\
\hline \multicolumn{3}{|l|}{ Idade (anos) } \\
\hline $20-29$ & 25 & 35,7 \\
\hline $30-39$ & 32 & 45,7 \\
\hline$\geq 40$ & 13 & 18,6 \\
\hline \multicolumn{3}{|l|}{ Estado civil } \\
\hline Solteiro & 44 & 62,9 \\
\hline Casado & 22 & 31,4 \\
\hline Viúvo & 3 & 4,3 \\
\hline Divorciado & 1 & 1,4 \\
\hline \multicolumn{3}{|l|}{ Procedência } \\
\hline Recife e região metropolitana & 58 & 82,9 \\
\hline Outros municípios & 12 & 17,1 \\
\hline
\end{tabular}

cientes declararam não fazer uso de profilaxia secundária à neurotoxoplasmose ou fazê-la de forma irregular.

Identificou-se também que $23(32,8 \%)$ pacientes tiveram o primeiro diagnóstico de AIDS nessa internação.

A contagem média de linfócitos T CD4 foi de 139,8 \pm 3,04 células $/ \mathrm{mm}^{3}$, com variação entre 1 célula $/ \mathrm{mm}^{3}$ a 714 células $/ \mathrm{mm}^{3}$. Dezesseis $(22,8 \%)$ pacientes apresentavam menos de 100 células T CD4+/ $\mathrm{mm}^{3}$. Quanto à carga viral, esta variou entre 1.110 cópias $/ \mathrm{mL}$ e 517.000 cópias $/ \mathrm{mL}$, com média igual a $137.080 \pm 39.380$ cópias $/ \mathrm{mL}$.

Quatro $(5,7 \%)$ pacientes apresentavam lesões cicatriciais de retinocoroidite, características de toxoplasmose ocular (Tabela 3). Em 1 (25\%) paciente, a lesão era periférica do tipo provável (Figura 1). Três (75\%) pacientes apresentavam lesões típicas, múltiplas, cicatriciais, sendo bilateral em um deles com presença de lesão macular e baixa importante da acuidade visual (Figura 2). Não se diagnosticou qualquer caso de retinocoroidite ativa, fosse ela típica ou provável.

\section{DISCUSS ÃO}

No presente estudo, encontrou-se baixa ocorrência de toxoplasmose ocular em pacientes com AIDS e neurotoxoplasmose $(5,7 \%)$. Na verdade, observaram-se apenas lesões cicatriciais, sem atividade. É possível que os pacientes tenham apresentado acometimento ocular assintomático ou oligossintomático que precedeu à neurotoxoplasmose. Segundo Cochereau-Massin et al., a toxoplasmose ocular precede a neurotoxoplasmose em aproximadamente metade dos $\operatorname{casos}^{(8)}$.

A neurotoxoplasmose em pacientes com AIDS deve-se à reativação de um foco cerebral inativo, decorrente da imunodeficiência celular ${ }^{(15)}$. Pacientes com um quadro de neurotoxoplasmose e AIDS não raro apresentam acometimento de outros sítios, particularmente pulmão, em até $26 \%$ dos $\operatorname{casos}^{(16)}$ e coração, em 3\% dos pacientes ${ }^{(17-18)}$. Seria de se esperar o acometimento ocular, pois existe um tropismo do taquizoíto por essa estrutura $^{(19)}$. Por outro lado, sabendo-se que a neurotoxoplasmose decorre de uma imunodeficiência que acarreta a reativação de cistos inativos no sistema nervoso central ${ }^{(20)}$, seria de se esperar que ocorresse reativação também em sítio ocular. No entanto, no olho de pacientes imunocomprometidos, talvez os mecanismos de imunidade ocular estejam preservados,

\begin{tabular}{|c|c|c|}
\hline Retinocoroidite & Frequência & Percentual (\%) \\
\hline \multicolumn{3}{|c|}{$\begin{array}{l}\text { Retinocoroidite cicatricial } \\
\text { provável }\end{array}$} \\
\hline Ausente & 69 & 98,6 \\
\hline Presente & 1 & 1,4 \\
\hline \multicolumn{3}{|c|}{$\begin{array}{l}\text { Retinocoroidite cicatricial } \\
\text { típica }\end{array}$} \\
\hline Ausente & 67 & 95,7 \\
\hline Presente & 3 & 4,3 \\
\hline
\end{tabular}


com relação ao $T$. gondii, mesmo diante da grave imunodeficiência observada na AIDS.

No ciclo parasitário do toxoplasma, o encistamento é um estágio essencial para sua manutenção no ambiente, fugindo do aparato celular endocítico que tende a degradar o material estranho intracelular(21). O olho não é um sítio preferencial de encistamento por dispor de dois mecanismos distintos, complementares, que geram condições inóspitas para o taquizoíta ${ }^{(22)}$.

Pesquisas experimentais comprovam que a resposta imune no olho de ratos imunocompetentes consiste em promover melhores condições de encistamento do taquizoíto,

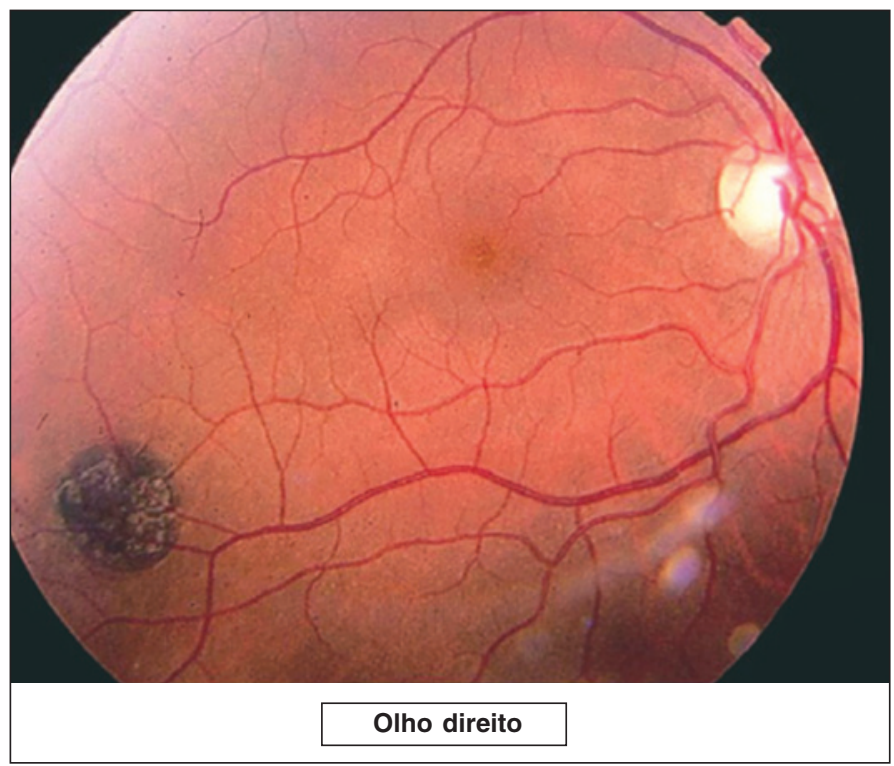

Figura 1 - Retinocoroidite provável toxoplásmica cicatricial em paciente com AIDS e neurotoxoplasmose por meio do aumento da síntese de interferon gama e de fator de necrose tumoral alfa ${ }^{(22)}$. Ao mesmo tempo, a quebra de barreira hematocular favorece acesso ao ambiente ocular de linfócitos T CD4+, T CD8+ e do epitélio pigmentar da retina, com propriedade fagocitária, aumentando a apoptose de células infectadas pelo aumento da expressão de MHC classe I, o que reduz a carga parasitária ocular e o aparecimento da lesão característica em atividade ${ }^{(22)}$.

Em ratos imunocomprometidos, o processo se inverte. Há redução da conversão de taquizoítos para a forma de replicação lenta de bradizoítos, por inibição da síntese de interferon gama e fator de necrose tumoral alfa, do que decorre maior carga parasitária na retina. No entanto, associada a esse fenômeno, há a depleção de linfócitos T CD4+ e T CD8+, que atuam no olho promovendo um nível menor de inflamação local e de apoptose, com consequente surgimento de lesões maiores e cicatriciais ${ }^{(23)}$.

Esses processos parecem permitir levantar a hipótese ser a imunodeficiência a possível causa de maior risco de desencadeamento da neurotoxoplasmose subsequente a uma toxoplasmose ocular, reduzido número de cistos e de lesões ativas em retina e presença de lesões extensas oculares, quando estas ocorrem em pacientes com AIDS.

O porcentual de pacientes com AIDS e neurotoxoplasmose para os quais foi identificada lesão cicatricial $(5,7 \%)$ foi menor que o relatado por outros pesquisadores, mas cumpre notar que ainda não há consenso quanto a essa taxa.

A literatura internacional refere que a toxoplasmose ocular em pacientes com AIDS é rara, acometendo $1 \%$ a $3 \%$ dos casos, no que difere do acometimento do sistema nervoso central ${ }^{(5,24)}$, mas essas pesquisas não contemplam pacientes com AIDS e neurotoxoplasmose.

Os dois únicos trabalhos ${ }^{(9,13)}$ localizados são retrospectivos e foram realizados em centro de referência de doenças
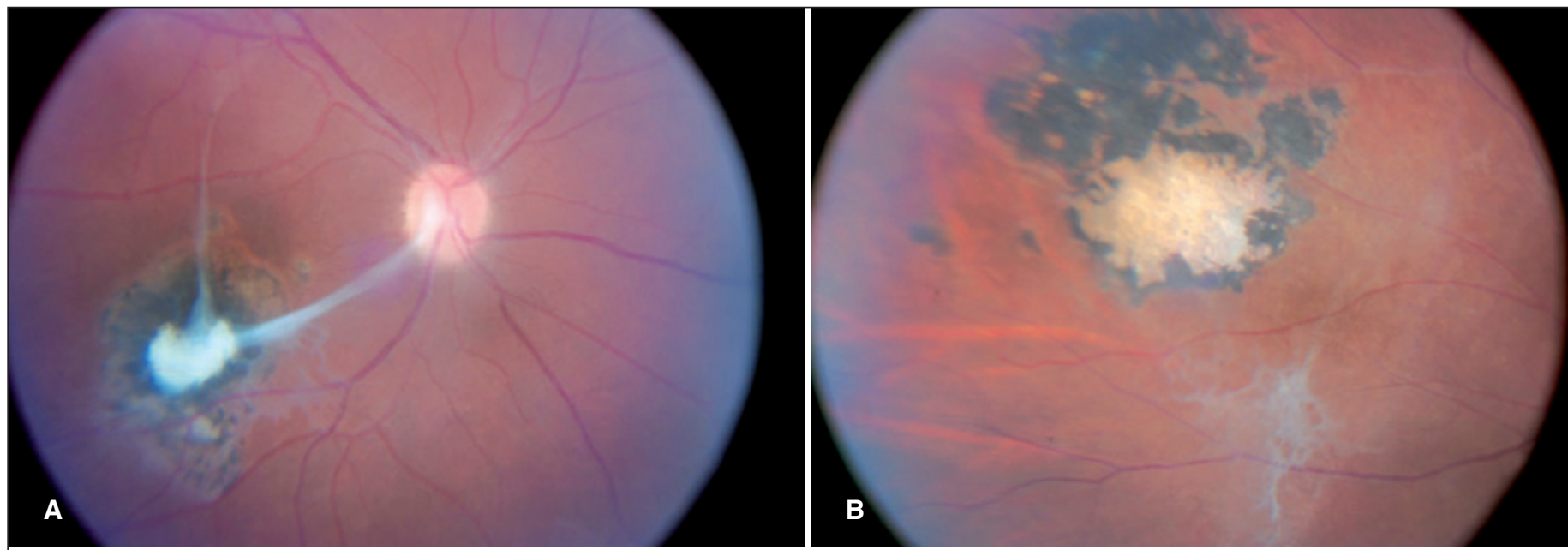

A= Olho direito: lesão macular, com tração vitreorretiniana; $B=$ Olho esquerdo: lesão periférica cicatrizada

Figura 2 - Retinocoroidite típica toxoplásmica cicatricial em paciente com AIDS e neurotoxoplasmose 
oculares em pacientes com AIDS, portanto podem ter incluído casos pouco usuais ${ }^{(9)}$, ao discutirem seus resultados. A explicação da ausência de lesões ativas dependerá de elucidação posterior no campo da imunologia. Os resultados desses autores ainda são motivo de pesquisas.

Diferente de outros autores, na presente pesquisa, não foram encontradas lesões oculares ativas em pacientes com AIDS e neurotoxoplasmose, mas a presença das lesões oculares cicatriciais parece indicar uma infecção passada por T. gondii, o que deve ser valorizado dado haver um risco aumentado de neurotoxoplasmose em caso de reativação da doença.

\section{ABSTRACT}

Purpose: Neurotoxoplasmosis is the most common central nervous system disorder in patients with AIDS. The occurrence of ocular toxoplasmosis in neurotoxoplasmosis is not well studied. The objective of this study was to investigate the occurrence of typical or probable toxoplasmic retinochoroiditis in patients with AIDS and neurotoxoplasmosis. Methods: A prospective case series was performed, including 70 patients of both genders, aged from 20 to 63 years, hospitalized in three public hospitals in Recife, Pernambuco, with such diagnosis according to the CDC criteria (1992), from January to October, 2008. Results: Patients were characterized by first neurotoxoplasmosis episode $(65,92.9 \%)$ or relapse $(5,7.1 \%)$, ignorance of AIDS diagnosis $(23,32.9 \%)$, mean CD4 T lymphocytes count of $139.8 \pm 3.04$ cells $/ \mathrm{mm}^{3}$ and mean viral load of $137,080 \pm 39,380$ copies $/ \mathrm{mL}$. All patients underwent ophthalmologic examination, consisting of ocular inspection, visual acuity measurement, investigation of ocular extrinsic muscle function and fundoscopy, using binocular indirect ophthalmoscope (model OHN 3.5 (Eyetec $^{\circledR}$ ) and 20 diopters external lens $\left(V^{2} k^{\circledR}\right)$. Four (5.7\%) patients presented retinochoroiditis scar lesions, characteristic of ocular toxoplasmosis, typical in $3(75 \%)$ of them and bilateral in one. There was no case of typical or probable active retinochoroiditis. Active ocular lesions were rare compared to scarring, which are associated with the presence of retinal cysts. Conclusion: Scarring lesions should be valued in patients with AIDS.

Keywords: Ocular toxoplasmosis; Acquired immunodeficiency syndrome; Eye/immunology

\section{REFERÊNCIAS}

1. Copeland R, Phillpotts BA. Ocular manifestations of HIV. eMedicine 2006:1-46.

2. Nussemblatt RB, Belfort Júnior R. Pathogenesis of inflammatory conditions of the retina: toxoplasmosis retinochoroiditis. In: Tabbara KF, Hyndiuk RA. Ophthalmology: infections of the eye. $2^{\text {nd }}$ ed. Boston: Little, Brown; 1996. p.61-5.

3. Hoffmann C, Ernst M, Meyer P, Wolf E, Rosenkranz T, Plettenberg A, et al Evolving characteristics of toxoplasmosis in patients infected with human immunodeficiency virus-1: clinical course and Toxoplasma gondii-specific immune responses. Clin Microbiol Infect. 2007;13(5):510-5.

4. Silveira C. Toxoplasmose: levantamento bibliográfico de 1997 a 2000. Arq Bras Oftalmol. 2001;64(3):263-70.

5. Jabs DA. Ocular manifestations of HIV infection. Trans Am Ophthalmol Soc. 1995;93:623-83.

6. Rodgers CA, Harris JR. Ocular toxoplasmosis in HIV infection. Int J STD AIDS. 1996;7(5):307-9.

7. Holland GN, O'Connor GR, Belfort Jr R, Remington JS. Toxoplasmosis. In: Pepose JS, Holland GN, Wilheimus KR. Ocular infection and immunity. Philadelphia: C.V. Mosby; 1996. p.183-223.

8. Cochereau-Massin I, LeHoang P, Lautier-Frau M, Zerdoun E, Zazoun L, Robinet $\mathrm{M}$, et al. Ocular toxoplasmosis in human immunodeficiency virusinfected patients. Am J Ophthalmol. 1992;144(2):130-5

9. Arevalo JF, Quiceno JI, García RF, McCutchan JA, Munguia D, Nelson JA, Fremann WR. Retinal findings and characteristics in AIDS patients with systemic Mycobacterium avium-intracellulare complex and toxoplasmic encephalitis. Ophthalmic Surg Lasers. 1997;28(1):50-4.

10. Cunningham ET Jr, Margolis TP. Ocular manifestations of HIV infection. N Engl J Med. 1998;339(4):236-44.

11. Moshfeghi DM, Dodds EM, Couto CA, Santos CI, Nicholson DH, Lowder CY, Davis JL. Diagnostic approaches to severe, atypical toxoplasmosis mimicking acute retinal necrosis. Ophthalmology. 2004;111(4):716-25.

12. Weiner A, BenEzra D. Clinical patterns and associated conditions in chronic uveitis. Am J Ophthalmol. 1991;112(2):151-8.

13. Zajdenweber M, Muccioli C, Belfort Júnior R. Acometimento ocular em pacientes com AIDS e toxoplasmose do sistema nervoso central: antes e depois do HAART. Arq Bras Oftalmol. 2005;68(6):773-5.

14. From the Centers for Disease Control and Prevention. 1993 revised classification system for HIV infection and expanded surveillance case definition for AIDS among adolescents and adults. JAMA. 1993;269(6):729-30.

15. Montoya JG, Liesenfeld O. Toxoplasmosis. Lancet. 2004;363(9425):1965-76. Review.

16. Melo HRL, Albuquerque VMG. Toxoplasmose. In: Melo HRL, Brito CAA Filho DBM, Souza SG, Henrique APC, Silva DB. Condutas em doenças infecciosas. Rio de Janeiro: Medsi; 2004. Cap.52. p.584-93.

17. Lanjewar DN, Agale SV, Chitale AR, Joshi SR. Sudden death due to cardiac toxoplasmosis. J Assoc Physicians India. 2006;54:244-5.

18. Eza DE, Lucas SB. Fulminant toxoplasmosis causing fatal pneumonitis and myocarditis. British. HIV Med. 2006;7(6):415-20.

19. Barragan A, Sibley LD. Migration of Toxoplasma gondii across biological barriers. Trends Microbiol. 2003;11(9):426-30.

20. Hegab SM, Al-Mutawa SA. Immunopathogenesis of toxoplasmosis. Clin Exp Med. 2003;3(2):84-105.

21. Black MW, Boothroyd JC. Lytic cycle of Toxoplasma gondii. Microbiol Mol Biol Rev. 2000;64(3):607-23. Review.

22. Wallace GR, Stanford MR. Immunity and Toxoplasma retinochoroiditis. Clin Exp Immunol. 2008;153(3):309-15.

23. Jones LA, Alexander J, Roberts CW. Ocular toxoplasmosis: in the storm of the eye. Parasite Immunol. 2006;28(12):635-42. Review.

24. Melamed J, Maestri M, Franco JA. Toxoplasmose ocular na Síndrome da Imunodeficiência Adquirida. Arq Bras Oftalmol. 1989;52(5):173-6. 